\title{
Role of funders in addressing the continued lack of diversity in science and medicine
}

\author{
Funders' goals for diversity, equity and inclusion are laudable, but they will only be successful if the funders \\ prioritize health disparities research and address biases in the funding application process.
}

\author{
Folakemi T. Odedina and Mariana C. Stern
}

$\mathrm{D}$ uring 2020 and 2021, the issues of diversity, equity and inclusion have risen to the top of the list of critical concerns that science and medicine faces. The senseless murders of George Floyd, Breanna Taylor and others at the hands of police officers in the United States, as well as racism and discrimination against Asians globally due to COVID-19 ${ }^{1}$, have led to monumental impact and clear realization of the racial injustice in every aspect of our lives. The lack of diversity, equity and inclusion in science and medicine is, however, not new. In fact, it has been seen as a prevalent epidemic, with bias, racism and discrimination at the core of the problem.

In response to the movements and events within the last year, most academic and scientific institutions feel the urge to, and are now scrambling, to right these wrongs, focusing on eliminating and preventing institutional racism and inequities in science and medicine. For example, the US National Institutes of Health (NIH) have affirmed their commitment to addressing structural racism in biomedical sciences, with support from President Joe Biden ${ }^{2}$. Other organizations have also issued statements and plans to do the same. Time will tell whether these efforts are sufficient and/or efficient in reducing inequities and promoting a culture of inclusion and representation.

Multiple barriers have been identified as impacting scientists from under-represented minority groups in biomedical research, including the lack of committed role models, ${ }^{3}$ inadequate mentoring ${ }^{4}$, insufficient preparation $^{3}$, low expectations ${ }^{3}$ and unfamiliarity with scientific culture and idioms ${ }^{3}$.

To address the problems of diversity, equity and inclusion in science and academia, it is important to focus on extramural funding in science and medicine, specifically the funding allocated to health disparities and diversity, equity and inclusion and also the funding success of researchers from under-represented minority groups. In the United States, these

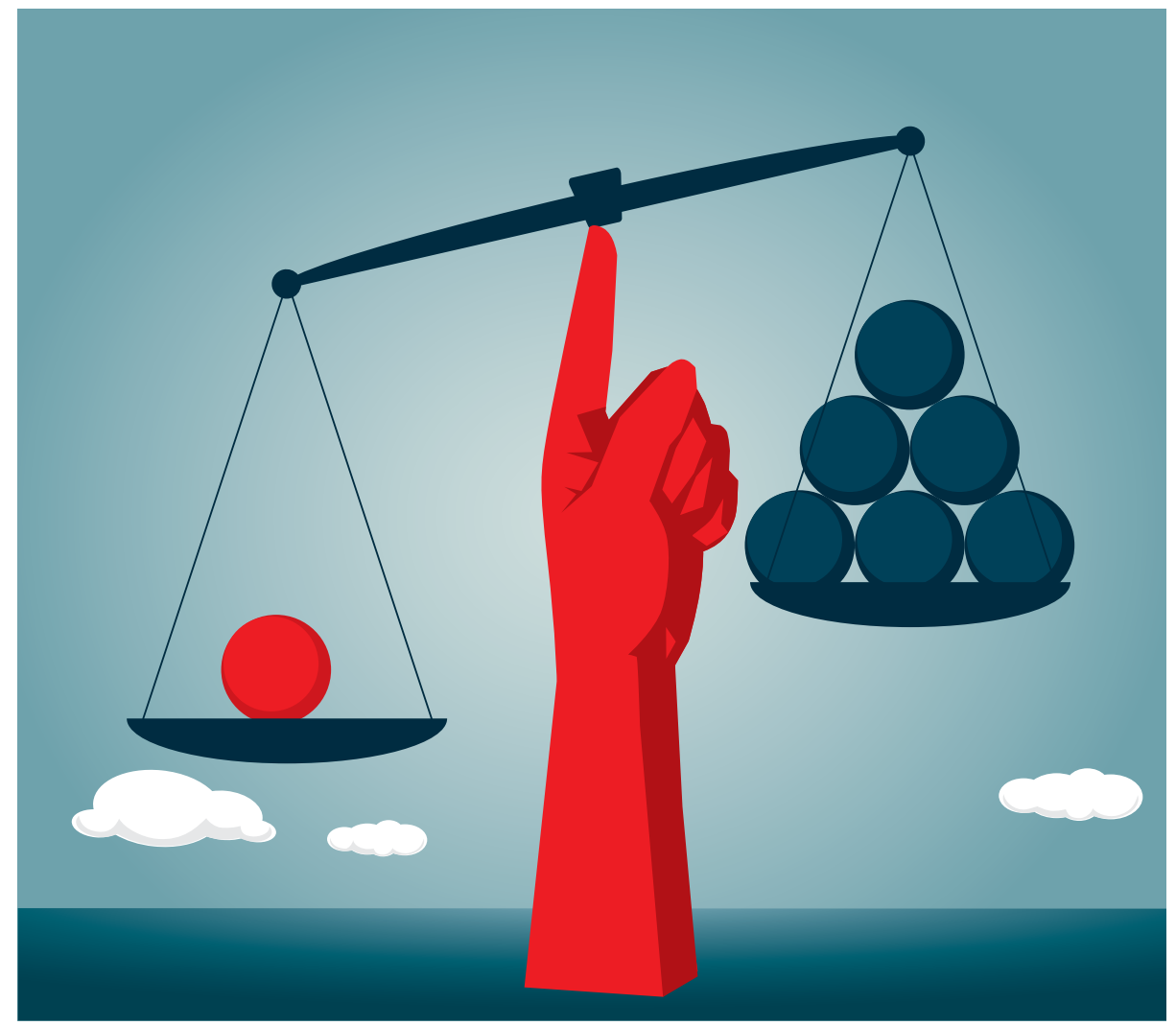

Credit: erhui1979 / DigitalVision Vectors / Getty

groups include Blacks or African Americans, Hispanics or Latinx, American Indians and Alaska Natives, and Native Hawaiians and other Pacific Islanders.

\section{Funding for health disparities}

Many scientists from under-represented minority groups focus their scholarship on research projects that address the needs of their communities, which may not be well aligned with the strategic priorities of their institutions or extramural funding agencies. Grant reviewers may question the significance of focusing research on minority populations if the health disparities are not glaringly obvious or dramatic, typically in comparison with the majority population. This perpetuates the disparity of research among underserved populations, along with the under-representation of minority groups in biomedical research. Lack of funding to support the research of under-represented minority investigators often leads them to change research interests to become more competitive, or cuts their research careers short.

Some of the reasons for the observed extramural funding disparities in science and medicine are funding agency priorities that may not be aligned with the research focus of scientists who are under-represented in science and medicine; competition with experienced, well-funded scientists who are often white; bias in the 
scientific review process; and a funding decision process that does not appropriately weigh the potential impact of awards and the importance of promoting the careers of Black, Latinx and Indigenous scientists.

Funding agencies need to do due diligence to ensure appropriate representation among program directors and scientific staff, which should include personnel from all races and ethnic groups to create responsive funding opportunities. Overall, a concerted effort must be made to prioritize career development to ensure the equal representation of minorities in the research workforce, including at funding agencies, in a proportion comparable to that in the general population. This effort needs to be made systematically across entire funding organizations and not limited to specific centers or efforts. This will facilitate the prioritization of research that benefits underserved communities as well as providing unique opportunities that will support workforce diversity. To this end, the $21^{\text {st }}$ Century Cures Act enacted by the US Senate and House of Representatives calls on federal funding agencies to prioritize policies for workforce diversity ${ }^{5}$.

\section{Competition for funds}

A growing trend that has become a significant threat to the funding of under-represented minority scientists is the attraction of already successfully funded non-minority scientists to health equity research, primarily to take advantage of increasing health disparity-focused funding opportunities.

Experienced investigators have generally benefited from increased federal funding over the years at the expense of new and early-stage investigators, who are mostly under-represented minorities and women ${ }^{6}$. There is additional concern that many grant-experienced white investigators with limited expertise in health disparities research specifically may fail to recruit or collaborate with under-represented minority investigators for their funded projects, which will further exacerbate disparities. Instead of competing, experienced investigators could collaborate with Black, Latinx and Indigenous scientists in applying for health disparity-focused grants as Multiple Principal Investigators or with the under-represented minority scientist as the Principal Investigator. This would provide a mutually beneficial opportunity for co-learning and mentoring.

Competition with experienced non-minority colleagues is compounded by the diversity-innovation paradox that is backed up by data from three decades of US doctorates across all scientific disciplines. The paradox is that whereas Black, Latinx or American Indian students, by gender or race, tend to produce more novel contributions, these innovations have lower impacts on their career trajectories compared to those of their peers, contributing to the lack of representation of minorities in leadership positions in science and medicine ${ }^{7}$. In other words, innovations coming from Black, Latinx or American Indian students are more likely to be discounted than if they come from white or Asian students.

\section{The scientific review process}

Although women and Latinx investigators have made some gains over the years, Black investigators continue to be underfunded. For example, Erosheva et al. ${ }^{8}$ reported a $45 \%$ gap between white and Black investigators in regard to NIH R01 awards between 2014 and 2016 in the United States.

A number of studies have reported bias during scientific review of grant proposals $^{6,9-12}$. Multiple reasons have been associated with this bias, including a perception that Black, Latinx or Indigenous investigators are less capable or less accomplished than white or Asian candidates. There is also perceived bias against minority-serving institutions (such as Historically Black Colleges and Universities and Hispanic-Serving Institutions) as not providing an appropriate environment for research.

The bias faced by Black scientists has led many to switch to academic administrative positions or leave academia altogether, as it is very difficult to be successful without extramural funding. In the United States, there is a nationwide problem in retaining Black scientists in academia. The domino effect of this is that the pipeline of Black scientists in academia and biomedical research continues to decrease, as upcoming trainees are discouraged by the experiences of Black scientists in academia, which are now widely shared through social media.

With a flawed scientific review process, the last opportunity to provide a fair and balanced review is the second-level review, when the funding decision is made (for example, the Council Review stage for US $\mathrm{NIH}$ funding and the Integration Panel Review for the Department of Defense (DOD) Congressionally Directed Medical Research Programs (CDMRP)). The second-level review typically provides a level of oversight to ensure that the scientific review is objective and the process complies with the policy of the funding opportunity.

The second-level review provides an opportunity for funders to weigh the potential impact of scored grants and make decisions to prioritize research that benefits underserved communities, enhances the training of scientists and fosters diversity of the biomedical workforce. An increasing number of funding agencies, including the NIH and DOD-CDMRP, are effectively using the second-level review to diversify their research portfolio. Of special note is the inclusion of consumer advocates (patient representatives or survivors) as part of the DOD-CDMRP Integration Panel, which has resulted in funding portfolios that are responsive to communities affected by health disparities and increased funding for investigators from these communities.

\section{Diversity tax}

There is also a growing impact of the so-called Diversity Tax, whereby a small number of scientists are burdened with significant committee assignments, including being asked to serve on committees for diversity, equity and inclusion and other taskforces, which takes time away from their research ${ }^{13}$. Given the limited number of researchers from minority backgrounds in research, the handful of such scientists serve on multiple committees.

Multiple studies across different environments have shown that we all win when the workforce is diverse ${ }^{14}$. In improving diversity, equity and inclusion in science and medicine, funding organizations must play a critical role and can contribute to improvements in the system. Funding organizations can address their own lack of representation among their staff, prioritize health disparities research at the time when they are developing funding opportunities, and make funding decisions that will address health disparities and promote scientists from under-represented groups. For example, funders can include specific review criteria that are focused on appropriate planning for the inclusion of health-disparity populations as well as on the diversity of the research team, including the lead investigators. Funding for health disparities should include scientists who represent the communities being served.

These efforts will only be successful if academic and scientific institutions, along with funding organizations, put significant efforts into not just recruiting scientists from under-represented minority groups, but also focusing on retention by supporting, mentoring and advancing the scientists towards long and successful careers. Immediate action is needed to stop the hemorrhaging of Black, Latinx and Indigenous scientists in science and medicine and to plug the leaks in the career development pipeline. 
Folakemi T. Odedina(D) ${ }^{凶}$ and

Mariana C. Stern²

${ }^{1}$ Division of Hematology/Oncology and Center for Health Equity and Community Engagement Research (CHCR), Mayo Clinic Florida, Jacksonville, FL, USA. ${ }^{2}$ Departments of Population and Public Health Sciences and Urology, Keck School of Medicine of USC, Norris Comprehensive Cancer Center, Los Angeles, CA, USA.

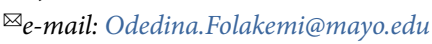

Published online: 11 November 2021

https://doi.org/10.1038/s41591-021-01555-8

\section{References}

1. Addo, I. Y. Soc. Sci. Humanit. Open 2, 100074 (2020).

2. Collins, F. S. et al. Cell 184, 3075-3079 (2021).

3. Jeste, D., Twamley, E., Cardenas, V., Lebowitz, B. \& Reynolds, C. III Am. J. Public Health 99, S31-S37 (2009).

4. Shavers, V. et al. J. Natl. Med. Assoc. 97, 1063-1077 (2005).

5. 21st Century Cures Act of 2016, Pub. L. 114-255. Approved 10 December 2016.

6. Nikaj, S., Roychowdhury, D., Lund, P. K., Matthews, M. \& Pearson, K. FASEB J. 32, 6410-6422 (2018).

7. Hofstra, B. et al. Proc. Natl Acad. Sci. USA 117, 9284-9291 (2020). 8rosheva, E. A. et al. Sci. Adv. 6, eaaz4868 (2020).

9. Ginther, D. K. et al. Science 333, 1015-1019 (2011).

10. Bhattacharjee, Y. Science 336, 969-970 (2012).

11. Kaatz, A. et al. Acad. Med. 91, 1080-1088 (2016).

2. Hoppe, T. A. Sci. Adv. 5, eaaw7238 (2019).

13. Trejo, J. Mol. Biol. Cell 31, 2752-2754 (2020).

14. Kamerlin, S. C. L. EMBO Rep. 21, e51994 (2020).

\section{Author contributions}

F.T.O. and M.C.S. both contributed to the writing of this Comment.

Competing interests

The authors declare no competing interests.

\section{The US National Institutes of Health approach to inclusive excellence}

The National Institutes of Health $(\mathrm{NIH})$ is committed to increasing the participation of underrepresented groups in science and medicine, through changes in our funding and recruitment processes. These actions provide a blueprint for the global research community.

\section{Marie A. Bernard, Alfred C. Johnson, Treava Hopkins-Laboy and Lawrence A. Tabak}

$\mathrm{D}$ iversity of thought and approaches is essential for maintaining creativity and innovation in any field ${ }^{1}$ and research shows that this is as applicable for science as it is for other fields ${ }^{2-6}$. The biomedical research ecosystem needs to take advantage of all creative minds to address the immense challenge of optimizing the health of the population (Fig. 1).

\section{Science and medicine still lack diversity} The US population is increasingly diverse ${ }^{7}$, but much of this population is untapped within the biomedical workforce. It is well known that some researchers are disadvantaged in receiving funding support through NIH R01 equivalent grants, ${ }^{8,9}$. Underrepresented groups (URGs), as defined by the National Science Foundation (NSF), are also underrepresented among program directors and principal investigators identified on NIH R01 equivalent grant applications and awards (Fig. 2).

The NIH has redoubled its efforts to enhance the participation of URGs in biomedical science, with an additional focus on those who may have experienced structural racism ${ }^{10-12}$. In an open letter in February 2021, the NIH Director Francis Collins wrote, "To those individuals in the biomedical research enterprise who have endured disadvantages due to structural

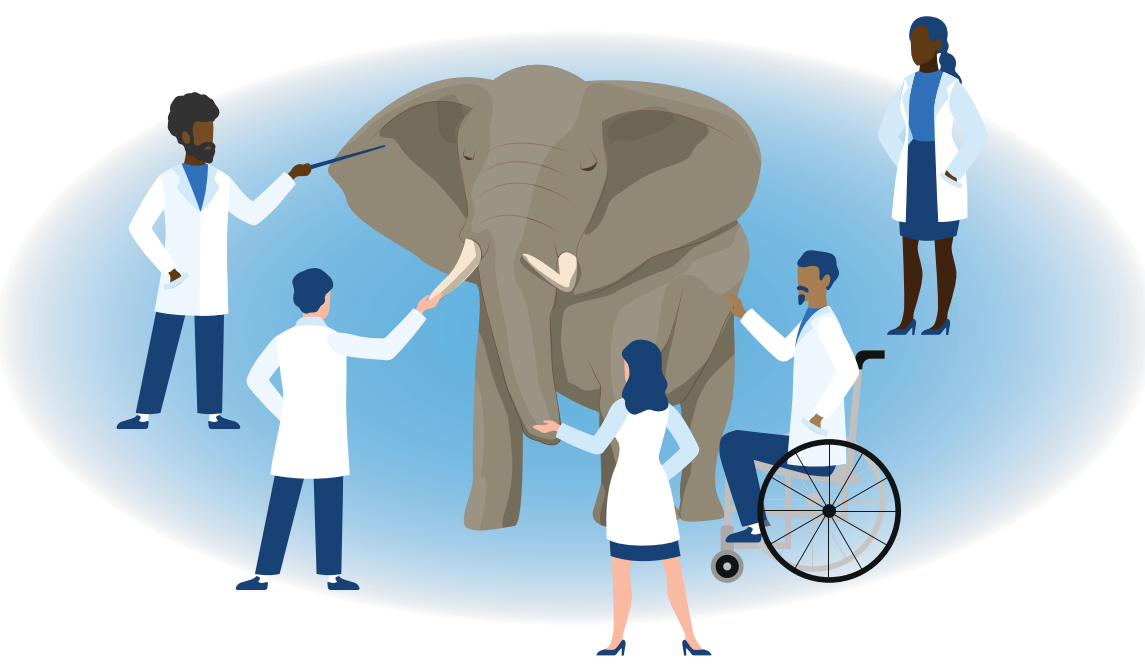

Fig. 1 | The challenge of biomedical research is akin to the examination of an elephant while lacking sight. The more diverse the input from several vantage points, the more likely the problem will be fully examined, and appropriate solutions derived. Art by ICF using imagery from Shuttlecock.

racism, I am truly sorry. NIH is committed to instituting new ways to support diversity, equity, and inclusion, and identifying and dismantling any policies and practices that may harm our workforce and our science" ${ }^{\prime 3}$.

Here we describe the NIH's efforts to foster equity in the biomedical research community, future funding implications and lessons that could be applied elsewhere in the world.

\section{The NIH UNITE initiative}

On 26 February 2021, the NIH publicly unveiled the UNITE initiative to address structural racism and the systemic barriers to the representation of URGs 